Revue d'histoire de l'Amérique française

REVUE D.HISTOIRE DE L'AMÉRIQUE FRANÇAISE

\title{
Les engagés pour le Canada au XVIII ${ }^{\mathbf{e}}$ siècle (suite et fin)
}

\section{Gaucher, M. Delafosse et G. Debien}

Volume 14, numéro 4, mars 1961

URI : https://id.erudit.org/iderudit/302082ar

DOI : https://doi.org/10.7202/302082ar

Aller au sommaire du numéro

Éditeur(s)

Institut d'histoire de l'Amérique française

ISSN

0035-2357 (imprimé)

1492-1383 (numérique)

Découvrir la revue

Citer cet article

Gaucher, M., Delafosse, M. \& Debien, G. (1961). Les engagés pour le Canada au XVIII ${ }^{\mathrm{e}}$ siècle (suite et fin). Revue d'histoire de l'Amérique française, 14(4),

583-602. https://doi.org/10.7202/302082ar d'utilisation que vous pouvez consulter en ligne.

https://apropos.erudit.org/fr/usagers/politique-dutilisation/ 


\section{LES ENGAGÉS POUR LE CANADA AU XVIII SIÈCLE * \\ par}

M. GAUCHER, M. DELAFOSSE et G. DEBIEN

(suite et fin)

Le 14 mai 1751, pour Québec, à François Macquet, capitaine de la Duchesse de Chaulnes, de Saint-Valery-sur-Somme, pour trois ans, à 300 livres de morue sèche, en tout, à 10 livres le quintal, soit à 30 livres pour leur service (Delavergne, notaire, liasse) :

[618] François Jouannet, 32 ans, garçon cordonnier, de La Rochelle.

[619] Jacques Gabaude, 21 ans, id. d'Albi.

[620] Jean Bétis, 26 ans, garçon cordonnier, de Tartas, en Gascogne, évêché de Dax. ${ }^{17}$ Signe.

Le 15 mai 1751, à Louis Pénisseau, négociant à Québec, retournant chez lui sur le Voltigeur de Saint-Valery, capitaine Vrague, aux mêmes conditions (Delavergne, notaire, liasse); Contrôle: 6 sols.

[621] Michel Chairtier, 21 ans, sans profession, de Châtillonsur-Indre. ${ }^{18}$ Signe.

Le 16 juin 1751, à Nicolas Brossard, capitaine du SaintUrsin, de La Rochelle, pour 3 ans, à 300 livres de sucre en tout, aller seulement. (Desbarres, liasse) :

[622] André Le Grot, 25 ans, cloutier, d'Angoulême. Signe.

[623] Jacques Franchisneau, 17 ans, cordonnier, de Poitiers.

Le 22 mai 1751, à André et Daniel Besse de la Barthe, frères, "négociants en compagnie » à La Rochelle, armateurs du Comte

*Voir notre Revue, XIII: 247-261, 402-421, 550-561; XIV: 87-108, 246-258, 430-440.

17 Chef-lieu de canton des Landes.

18 Chef-lieu de canton de l'arrondissement de Châteauroux (Indre). 
de Jouy, capitaine Guillot (Chameau, notaire, liasse) (Contrôle: 36 sols pour les trois) :

[624] Jean de Saint-Martin, 25 ans, tailleur, de Cognac. Signe.

[625] Nicolas Barbot, 18 ans, perruquier, $i d$. $i d$.

[626] Jacques Mesneau, 22 ans, tailleur, de Saintes, qui pendant trois ans iront travailler de leur métier et qui l'apprendront à des apprentis. Le salaire est de 50 livres par an. Ils seront nourris jusqu'à leur embarquement, et en Nouvelle-France ils le seront « au pays et viande du pays, ainsi que les engagés ont accoutumé de l'être audit Canada».

Le $1^{\text {er }}$ et le 7 juin 1751 , pour Québec, aux mêmes conditions, Etienne Ranjard, négociant à La Rochelle, armateur du SaintThomas, capitaine Martin Larreguy (même notaire) :

[627] Henri Hervie, 20 ans, tailleur d'habits, de Ganat, ${ }^{19}$ en Bourbonnais.

[628] Jean Teil, 25 ans, boulanger, de Marsillac, en Angoumois. ${ }^{20}$

[629] Jean Tounoir, 16 ans, tailleur d'habit, de Thouars, en Poitou. Signe.

$\mathrm{Au}$ P. Maurice Meignée, de l'ordre de Saint-Jean-de-Dieu de la Charité et supérieur du couvent de Louisbourg, de présent à La Rochelle, s'engagent le 9 juin 1751, trois hommes «sans profession » mais qui auront d'assez beaux salaires. Contrôle 30 sols en tout. (Delavergne, liasse) :

[630] Louis Coustard, 22 ans, de Pons-l'Abbé en Saintonge. ${ }^{21}$ 180 livres.

[631] Jean Geoffrin, 26 ans, de Brie-sur-Matha. ${ }^{22} 180$ livres. Signe.

[632] François Sorin, 15 ans, de Saint-Maixent..23 100 livres.

${ }^{19}$ Chef-lieu de l'arrondissement de l'Allier.

20 Marcillac-Lanville, commune du canton de Rouillac, arrondissement d'Angoulême (Charente).

21 Commune du canton de Saint-Porchaire, arrondissement de Saintes (Charente-Maritime).

22 Commune du canton de Matha, arrondissement de Saint-Jean d'Angély (Charente-Maritime).

23 Chef-lieu de canton des Deux-Sèvres. 
En 1753 les capitaines reviennent aux salaires comptés en sucre ou en morue sèche.

Le 6 avril Frédéric-François de Coulleri, capitaine de $l a$ Charmante Victoire de La Rochelle, appartenant à J. B. Soumbrun, engage pour Québec, à 300 livres de sucre brut (Desbarres, notaire) :

[633] Jean-Baptiste Ledoux, 24 ans, menuisier, de Beauvais en Picardie.

[634] Eustache Rousseau, 22 ans, menuisier, de Landrecies.. ${ }^{24}$ Signe.

Le 14 avril, à Nicolas Brossard, capitaine du Triton, de La Rochelle, appartenant à J. B. Soumbrun, pour Québec (même notaire) :

[635] Joseph Hardy, 27 ans, menuisier, de Vannes en Hainault. Signe.

[636] Claude Burreau, 23 ans, menuisier, de Moulins, qui signe.

[637] François Langevin, 20 ans, menuisier, d'Angers, qui signe.

A Charles Boucher, capitaine du Solide, de $300^{\text {tx }}$, toujours à 300 livres de sucre :

\section{Le 25 mai}

[638] Pierre Pascaud, 19 ans, garçon boucher, de Saintes.

le 28 mai

[639] Antoine Claimanceau,, 22 ans, garçon boulanger, de Prédelois ? en Aunis. ${ }^{25}$

[640] Antoine Nadault, 36 ans, de Chague, ${ }^{26}$ en Angoumois.

Le 23 juillet 1758 , à Robert de Seille, capitaine de la Société de Calais, de $95^{\text {tx }}$, affrété par le roi (Tardy, notaire, liasse) :

[641] Pierre Ballay, 27 ans, ci-devant employé dans les fermes du Roi, demeurant à La Rochelle, mais de la paroisse de

24 Chef-lieu de canton de l'arrondissement d'Avesnes (Nord).

25 Sans doute Predd'Ouaille, commune d'Esnandis, canton de La Rochelle (Charente-Maritime).

26 Non identifié. 
Brossac en Saintonge, ${ }^{27}$ qui signe. Il ira à Louisbourg ou «autre endroit de l'île Royale », où il sera nourri, entretenu, habillé et chauffé pendant trois ans. A la fin: 3 quintaux de morue sèche. Pas de retour. Droit de contrôle: 6 sols.

Le 24 juillet 1753,

[642] Marie Renault, 35 ans, servante, née à La Rochelle, paroisse Notre-Dame, s'engage au Sieur Joseph Lartigue, bourgeois, demeurant à l'île Royale; pour le servir comme domestique pendant 3 ans, à 90 livres par an. Aller seulement.

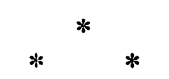

Louisbourg est désormais le centre vers où sont dirigés les engagés. On a l'impression que les capitaines de navire perdent le contrôle de ce mouvement. Les hommes de l'île Royale, les entrepreneurs de travaux, les Congrégations religieuses s'entendent avec des négociants, avec des armateurs de confiance plutôt qu'avec des capitaines. Du même coup, les conditions de passage et les salaires sont améliorés. La guerre est proche. Il faut des hommes.

Le 26 mars 1754, à Pierre Hébert, capitaine de la goëlette l'Echappé de Louisbourg, de 60 à $70^{\text {tx }}$, s'engagent pour 3 ans, à 550 livres: 100, 150 et 200 livres par an (Tardy, not. liasse) :

[643] Jacques Michel, 20 ans, apprenti chaisier, de la paroisse Saint-Pierre-de-Poitiers.

[644] Guillaume Marre, 21 ans, garçon perruquier, de Druelle en Rouergue, ${ }^{28}$ évêché de Rodez, qui signe. Droit de contrôle, 4 livres, 16 sols.

Pour le compte du sieur Couret et compagnie, entrepreneur de fortifications à Louisbourg, Michel Rodrigue engage le 20 avril 1754 :

[645] Jacques Ganeray, 30 ans, forgeron, de Viré, province du Maine, ${ }^{29} 3$ ans. Non seulement il ne travaillera que de son métier mais il sera nourri à pain de France et aura le petit coup d'eau-de-vie chaque matin, 180 livres par an

27 Commune du canton de Barbezieux (Charente).

28 Druelle, commune du canton de Rodez (Aveyron).

29 Viré-en-Campagne, commune du canton de Brûlon (Sarthe). 
et le retour. Il fera le voyage sur le Pontchartrain, capitaine Jean Frément. Signe (Desbarres, notaire, liasse).

le 8 mai

[646] Pierre Indreau, 30 ans, charpentier de gros œuvre, de la paroisse Notre-Dame de La Rochelle où il est né, pour 3 ans, à 60, 80 et 90 livres par an; 24 livres d'avance pour s'équiper. Contrôle: 12 sols (Tardy, not. liasse).

Le 8 mai et le 6 juin 1753 Rodrigue pourvoit de deux engagés la veuve Lartigue, bourgeoise de Louisbourg, aux conditions désormais ordinaires de 40, 50 et 60 livres par an (Tardy, not. liasse) :

[647] Jean Belliard, 14 ans, garçon voilier, de Saint-Sauveur de La Rochelle, en présence d'Anne Marot, sa mère, qui est veuve de Jean Belliard, cuisinier, et qui habite avec son fils - un autre fils sans doute - paroisse Notre-Dame à La Rochelle. Contrôle, 12 sols.

[648] François Charreau, dit Belliard, 20 ans, de Saintière ${ }^{30}$ près Saint-Maixent en Poitou. Contrôle: 12 sols.

Ces deux noms de Belliard n'établissent-ils pas un lien entre ces deux hommes, qui sont peut-être parents, ou ont fait voyage ensemble?

Etienne Ranjard est l'homme des religieux de l'hôpital de la Charité de Louisbourg. Il leur trouve 5 hommes (Tardy, not. liasse) :

le 24 avril 1754

[649] François Renaudeau, 28 ans, laboureur à bras, de Tangonla-Ronde, en Aunis. Il partira sur le Pontchartrain de La Rochelle, capitaine Remont. 100 livres par an, 30 d'avance. Signe. Contrôle: 24 sols.

le 20 mai

[650] Pierre Millet, 28 ans, cuisinier, de la paroisse de NotreDame-du-Havre, pour 3 ans, en qualité de cuisinier, à 200 livres par an, 90 d'avance. Aller et retour. Il partira

30 Hameau de la commune de Vernoux-en-Gâtine, canton de Secondigny, arrondissement de Parthenay (Deux-Sèvres). 
sur l'Elisabeth, de La Rochelle, capitaine Neau. Contrôle: 48 sols.

[651] Isaac Sabourain, 35 ans, tireur d'étain, de Saint-Saturnin de Saint-Maixent.

[652] Isaac Barraut, 35 ans, tireur d'étain, de Saint-Saturnin de Saint-Maixent, à 90 livres par an, 40 d'avance.

le 5 juin

[653] Sébastien Perrot, 22 ans, journalier, de Fointaine-LaGuyon, près Chartres, ${ }^{31}$ à 80 livres par an, 40 d'avance. Contrôle: 12 sols.

Le 19 août 1754, à Pierre Sorin, capitaine de la Diligente, pour 3 ans, à 30,40 et 50 livres par an (Delavergne, notaire), pour l'île Royale :

[654] Pierre Maria, 19 ans, tailleur d'habits, de Saint-Jean-deLiversay, en Aunis. ${ }^{32}$

[655] Antoine Maria, son frère, 18 ans, du même métier.

En 1755, quatre engagés partent pour Québec, et quatre pour Louisbourg. Pour Québec, Denis Goguet, négociant à La Rochelle, pour son compte le 12 février; à 300 livres de «tabac du cru du pays » (Tardy, notaire, liasse) :

[656] Pierre Manceau, 37 ans, charpentier de gros œuvre, de Saint-Briec au Maine, ${ }^{33}$ qui habite La Rochelle.

[657] Jean Boutet, 24 ans, charpentier de gros œuvre aussi, de Var en Angoumois, ${ }^{34}$ qui habite La Rochelle.

Le 20 mars, il est plus généreux, mais il recrute alors pour $\mathrm{M}^{\mathrm{e}}$ Estèbe, conseiller au Conseil Supérieur de Québec, qui a dû lui laisser des instructions.

[658] Jean Roy, 28 ans, jardinier, de la paroisse de la ChapelleTiré, province de Gâtine, au diocèse de La Rochelle, ${ }^{35}$

31 Fontaine-La-Guyon, commune du canton de Courville-sur-Eure, arrondissement de Chartres (Eure-et-Loir).

32 Commune du canton de Courçon, arrondissement de La Rochelle (Charente-Maritime).

33 Commune du canton de Grez-en-Bouère, arrondissement de ChâteauGonthier (Mayenne).

34 Commune du canton de Saint-Amant-de-Boixe, arrondissement d'Angoulême (Charente).

35 Commune du canton de Coulonges-sur-l'Autize, arrondissement de Niort (Deux-Sèvres). 
pour 3 ans, à 150 livres par an, 50 livres d'avance. Aller et retour. Signe. Contrôle: 30 sols (Tardy, notaire, liasse).

Aussi pour Québec, Charles Vincelot, capitaine du Solide de La Rochelle, appartenant à J. B. Soumbrun, négociant, recrute (Desbarres, notaire, liasse) le 23 avril :

[659] Pierre Petel, 18 ans, cuisinier, natif de Mouchies au diocèse de Beauvais, ${ }^{36}$ aller seulement, 300 livres de sucre brut. Signe.

Les départs pour Louisbourg se font dans une saison plus avancée et tous les contrats sont passés par devant Tardy:

Le 2 juillet, à Jean Lambert, capitaine du Duc Duescar de Saint-Valery-sur-Somme, armé par Louis Perdriau, négociant à La Rochelle:

[660] Gabriel Thibault, 30 ans, garçon boulanger, de Blois, qui demeure depuis six semaines à La Rochelle chez le sieur Marillet en la paroisse Notre-Dame. Il n'obtient que l'aller, et pour ses trois ans 100 livres de morue sèche. Signe. Contrôle: 6 sols.

A Lartigue, aîné, négociant à Louisbourg, le 30 juillet 1755, par l'entremise de Michel Lecomte, capitaine du Juste, qu'arme Michel Rodrigue; pour 3 ans, à 40,60 puis 80 livres par an, 15 d'avance:

[661] Jean-Baptiste Suire, 30 ans, maître tonnelier, de NotreDame-de-La-Rochelle, qui signe. Contrôle: 6 sols.

[662] Pierre Normand, 21 ans, garçon cordonnier, de Vars (?) ${ }^{37}$ près Barbezieux. Contrôle : 6 sols.

Le 13 août 1755, à Claude Morin, négociant de Louisbourg, alors embarqué sur l'Heureux, capitaine Boubaillie:

[663] Jean Chausset, 14 ans, domestique, de Marencennes, ${ }^{38}$ paroisse de Surgères, 3 ans, 40 livres par an.

(Oise).

36 Mouchy-le-Châtel, canton de Noailles, arrondissement de Beauvais

37 Commune du canton de Saint-Amant-de-Boixe, arrondissement d'Angoulême (Charente).

38 Saint-Germain-de-Marencennes, canton de Surgères, arrondissement de La Rochelle (Charente-Maritime). 


\section{VI - Guerre de Sept Ans}

\section{(1756-1763)}

Avant la déclaration officielle de la guerre, qui suivait un long état de lutte, Michel Rodrigue est à La Rochelle le seul homme qui lève des engagés. Ils partent sur son navire les Deux Frères, capitaine Duficharet, qui fait voile. Ces engagés n'ont que 3 quintaux de morue à 15 livres, à la fin de leur service. (Tardy, notaire, liasse) :

\section{le 14 avril 1756}

[664] Charles Busseraud, 39 ans, boulanger, né et demeurant à Québec. Contrôle: 6 sols.

[665] François Thibaud, 29 ans, garçon cordonnier, de SaintMaurille d'Angers, mais demeurant à La Rochelle, paroisse Saint-Sauveur (id.).

[666] Pierre Lavallerie, 23 ans, de la paroisse Saint-Jean-deLyon, garçon cordonnier, demeurant à La Rochelle, paroisse Saint-Sauveur.

Point d'engagés en 1757 , et 1758 est la dernière année où l'on trouve des engagements pour le Canada à La Rochelle. C'est l'année de la prise de Louisbourg.

Les 3 et 9 février 1758, le Père Prieur de l'hôpital de Louisbourg, toujours par la voie d'Etienne Ranjard, engage pour 80 livres par an, mais sans le retour:

[667] Edme Brossier, dit Chateauneuf, 29 ans, boulanger de Chateauneuf en Bourgogne. ${ }^{39}$

[668] François Muret, 19 ans, garçon chirurgien, de Grammont, pays de la Marche. ${ }^{40}$ Qui signe.

Le 15 mars 1758, Etienne Coindet, capitaine de la Fille Unique, prend pour Québec, trois hommes levés par François

39 Commune du canton de Puilly (Côte d'Or).

40 Grand-Mont, commune de Clugnat (Creuse). 
Gazan, négociant à La Rochelle, à 300 livres de morue à 10 livres le quintal. Ces hommes demeurent à La Rochelle. (Tardy, liasse) :

[669] François Lafond, 28 ans, garçon tailleur d'habits, de la paroisse de Mont-de-Marsan, évêché d'Aires.

[670] Louis Berte, 28 ans, garçon cordonnier de Rambouillet en Beauce.

[671] Similien Gendron, 25 ans, garçon cordonnier, de la paroisse de Saint-Sébastien de Nantes.

Le 21 avril 1758, pour Québec, François Mitteau, capitaine du Grand Gédéon, de La Rochelle, armé par Michel Rodrigue (Tardy, notaire, liasse), aux mêmes conditions, partent trois ouvriers qui travaillent à La Rochelle:

[672] Jacques Desjean, 19 ans, tailleur d'habits, de la ville épiscopale de Mirepoix, paroisse Saint-Martin.

[673] Pierre Fouissard, 23 ans, garçon cordonnier, de Poitiers, paroisse Notre-Dame.

[674] Pierre Médard, 24 ans, garçon cloutier, de Poitiers, paroisse Saint-Porchaire.

Louisbourg tombe aux mains des Anglais le 27 juillet 1758 après un long siège. Le 18 octobre, [675] Madeleine Bloc, âgée de 24 ans, native de Louisbourg, de présent à La Rochelle, déclare devant Tardy (liasse) que «depuis la prise faite par les Anglais dudit lieu de Louisbourg et dès le 7 août dernier 》 elle «s'est engagée à dame Joseph Guyon, épouse de Monsieur de Tournay, ci-devant officier de ladite colonie, pour la servir pendant une année en qualité de fille de chambre, qui a commencé ledit jour sept août dernier ». Elle veut sans doute par là avoir une preuve qu'elle n'a pu rejoindre sa maîtresse, et faire établir que ses gages de 30 livres lui sont cependant dus pour l'année commencée. 
592

REVUE D'HISTOIRE DE L'AMÉRIQUE FRANÇAISE

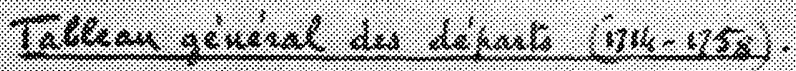

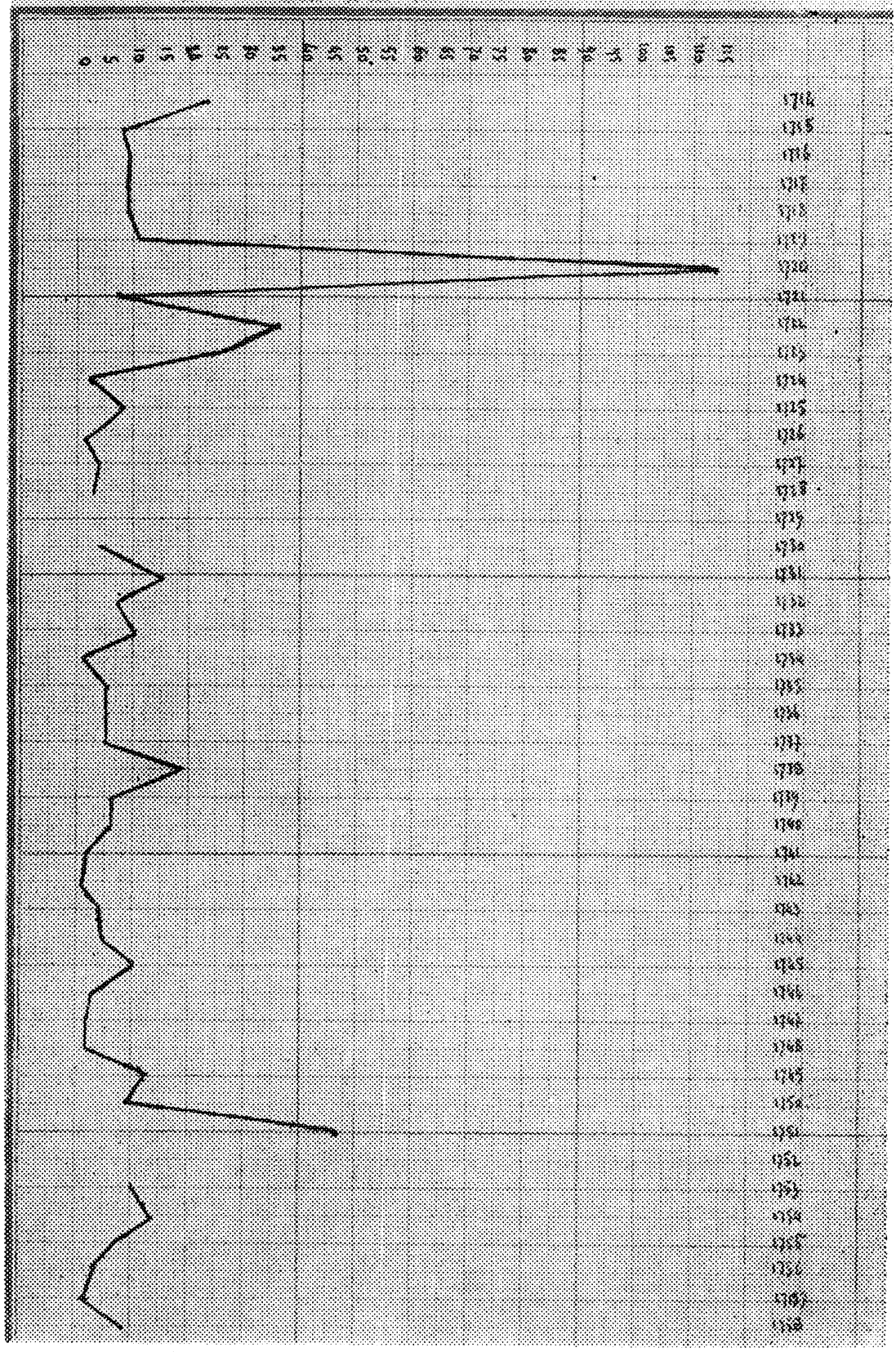




\section{CONCLUSION}

Ces 496 noms d'engagés ${ }^{41}$ ne font pas une foule et répartis sur près d'un demi-siècle ils ne représentent pas un mouvement qui pût attirer l'attention des contemporains.

Sur ces 44 années - 1714-1758 —, dont deux sans départ, 27 ont dix, ou moins de dix engagés. C'est donc un filet d'émigration, comme le montre le tableau général des engagements. Sans doute il faut redire que ce sont là non des chiffres certains, mais ceux que donnent les documents et faire la part des lacunes des minutes. La ligne générale du mouvement n'en apparaît pas moins avec une suffisante probabilité. $\mathrm{Au} \mathrm{XVIII}{ }^{\mathrm{e}}$ siècle on ne part plus guère pour le Canada avec un contrat d'engagement.

Quand, par trois fois en 1720 , en 1722 et en 1751 le mouvement prend plus d'ampleur, le nombre des contrats montant ces années-là à 114 , à 35 et à 45 , c'est sous l'action d'une pression extérieure, c'est la propagande qui agit. Les efforts d'une compagnie de colonisation en 1720, ceux du gouvernement en 1751, donnent alors aux départs un tour accéléré. Dès que les établissements projetés sont ébauchés et la propagande éteinte, on retrouve les petits nombres du train ordinaire.

$\mathrm{Au}$ surplus qu'elle se réduise à quelques hommes par an ou qu'elle monte à une centaine d'engagés cette émigration reste une émigration dirigée. Elle paraît obéir avant tout à des préoccupations administratives et politiques. Les 2 ou 3 ou 4 ou 6 engagés des années communes correspondent eux aussi aux ordres du roi, à la surveillance des Amirautés qui obligent tout navire qui fait voile vers les colonies, à embarquer un nombre d'engagés proportionné à son tonnage. Ce souci est ancien. Il est devenu un usage. En continuant d'imposer l'embarquement de quelques engagés les bureaux de la Marine rêvent encore d'un peuplement automatique et peu coûteux à l'État.

Le désir du gouvernement est aussi de renforcer des zones délicates ou litigieuses par un peuplement stable de cultivateurs et de marins. Il veut contrecarrer les ambitions britanniques,

${ }^{41}$ On ne tient pas compte ici des 179 noms relevés dans les registres paroissiaux de l'île Saint-Jean. 
peut-être préparer une reprise, et appuyer des revendications territoriales. L'Etat veut grossir un mouvement que les grandes distances, les difficultés d'un climat de mauvaise réputation ont réduit à rien. A côté d'une émigration administrative il s'agit d'une émigration stratégique. Il n'y a pas à parler des hauts et des bas d'un appel colonial. Rien ici de très spontané. Les variations sont des poussées, puis des retombées, imputables au manque de persévérance, aux hésitations du gouvernement plutôt qu'au changement des conditions politiques.

Comme les contrats de passage de colons libres sont d'autre part très rares dans les minutes du XVIII ${ }^{\mathrm{e}}$ siècle, on est devant cette conclusion: ou il n'est plus d'usage de signer un accord écrit avec le capitaine ou l'armateur quand on est un passager libre, ou les liens démographiques du Canada avec la métropole sont devenus si lâches que la colonie ne doit compter pour grandir que sur elle-même. Une tradition d'émigration - comme celle qu'on voit vers les Antilles -- ne s'est pas établie vers la Nouvelle-France, qui ne passe pas pour un pays aux fortunes rapides.

Si on peut parler d'émigration dirigée on peut parler aussi d'émigration jusqu'à un certain point choisie. Il en avait été un peu de même au XVII ${ }^{\mathrm{e}}$ siècle, au moins dans la mesure où les gens du roi et les congrégations religieuses, Récollets, Jésuites, prêtres de Saint-Sulpice, Ursulines et Hospitalières avaient fait lever des hommes pour leur service et leurs seigneuries. Peu ou point d'endettés parmi ces recrues du XVIII ${ }^{\mathrm{e}}$ siècle. Du moins aucun acte de reconnaissance de dettes ne précède les contrats d'engagement, comme on le voit au XVII ${ }^{e}$ siècle.

L'examen des métiers de ceux qui partent prouve que l'on n'a pas mené au Canada n'importe qui. Sur les 496 engagés dont les contrats nous donnent les noms, 63 seulement sont de métiers inconnus ou trop jeunes pour avoir déjà commencé un apprentissage quelconque. De ces 433 engagés à la profession précise, la date de départ, le nom de l'engagiste, et mieux encore les conditions de l'engagement, permettent de faire deux groupes bien distincts, d'importance d'ailleurs fort inégale. D'un côté les hommes qu'on demande et dont le métier a déterminé le départ; de l'autre les hommes dont le métier n'était pas demandé. 
Sont apparemment parmi ces derniers tous les journaliers et « garçons de service », ou ceux que les capitaines désignent ainsi. Il en est 59 , tous au salaire de 300 livres de sucre brut ou de morue, qui est un salaire minimum. Il faut croire que la plupart d'entre eux ne sont pas ces gens à tout faire que disent les capitaines, mais comme ils peuvent être mis aux divers gros travaux des champs et des bois, il a paru plus expédient aux engagistes de leur donner le nom de gens de service. Ainsi ils seront peut-être cédés plus facilement. C'est la piétaille de cette émigration.

On distingue les domestiques des gens de service. Les domestiques sont gens de maison, comme les servantes. Les unes et les autres sont peu nombreux: 4 servantes, 3 domestiques.

Les laboureurs à bœufs ou à bras, qui sont au nombre de 53, les 15 jardiniers, sont hommes de la terre et qui resteront à la terre. Ils ont été assurément recrutés pour les travaux agricoles.

Les 30 tailleurs d'habits ont dû être demandés. Remarquons leur groupement, ainsi que celui des cordonniers au nombre de 23. Il paraît aussi hors de doute que tous les ouvriers du bois qui vivent plus ou moins loin des coupes, les 27 tonneliers, les 13 charpentiers, les 9 menuisiers, les charbonniers, les 6 scieurs de long, les plieurs de cercles, sabotiers, fendeurs de bois, tailleurs de roues, charrons, les 3 couvreurs, sont venus parce qu'au Canada on avait besoin d'ouvriers de ces métiers, ainsi que d'ouvriers du fer et métaux: 2 forgerons, 2 chaudronniers, 5 taillandiers, 2 armuriers, 3 potiers d'étain, serruriers, 6 cloutiers.

Si on voit trois boulangers partir le même jour, 31 mars 1720, et deux fariniers le 2 avril 1720, c'est qu'ils savent que le travail ne leur manquera pas. Si nous en doutions, il suffirait d'observer leurs salaires et leurs conditions qui ne sont pas celles des engagés à 300 livres de sucre pour trois ans. Il est 18 boulangers et 11 fariniers. De même le groupement des perruquiers laisse croire qu'on les appelle.

On hésite devant les 12 tisserands, les sergetiers, les 7 cardeurs ou tireurs de laine, les bonnetiers, soquetiers, boutonniers, gantiers et ouvriers en soie. Tous ces ouvriers du textile sont 
infiniment moins nombreux à passer l'océan qu'au siècle précédent. L'industrie textile rurale n'aura pas eu à souffrir de crises aussi profondes qu'au XVII ${ }^{e}$ siècle. Ces tisserands, ces sergetiers sont des isolés et rien n'assure qu'ils travailleront de leur métier en Nouvelle-France, et la conclusion, indirecte mais très ferme, est que les groupes serrés de tisserands et sergetiers qui quittent nos campagnes de l'Ouest au $\mathrm{XVII}^{\mathrm{e}}$ siècle sont poussés à sortir par une crise de l'industrie textile, tandis que le nombre très réduit de tisserands correspond au XVIII ${ }^{e}$ siècle aux besoins d'une colonie paysanne où bien des hommes doivent savoir manier la navette.

Le vigneron, le cordier, le musicien, l'orfèvre, l'écrivain, le matelassier, les deux tapissiers, le pâtissier, les deux teinturiers et les 6 cuisiniers, le cocher et l'ouvrier en cire, ne sont-ils pas des hommes qui simplement cherchent du travail ? Mais les six chirurgiens, la sage-femme, les matelots, les voiliers, les tanneurs, les ouvriers du bâtiment (15 maçons, 3 tailleurs de pierre, 2 vitriers) sont recrutés pour des travaux précis.

L'impression générale est donc que le mouvement des engagés vers le Canada au XVIII' siècle n'est pas un recrutement de hasard. Il révèle que la misère et la mendicité ne sont pas aussi grandes dans la France de la première moitié du XVIII ${ }^{\circ}$ siècle qu'au siècle précédent. Les conditions de travail contraignent moins directement les pauvres gens à gagner les pays lointains, prometteurs de pain et de sécurité. L'attrait de millions d'arpents à défricher ne joue plus autant qu'au XVII ${ }^{e}$ siècle ni les traditions de mobilité paysanne qui tenaient sur les chemins à la merci des embaucheurs de toutes sortes tant de pauvres hères et de sans travail.

Quelles étaient donc les conditions de vie qui décidèrent ces hommes à partir ? S'il semble bien qu'on doive écarter l'hypothèse de crises économiques précises, ne faut-il pas en revanche s'arrêter à des conditions sociales particulières ? On devine que les effets de la propagande qui présida au recrutement brouillent les pistes et obscurcissent la réponse, car nous ne sommes pas devant un mouvement spontané. Toutefois nous avons deux commencements d'éclaircie par l'examen des contrats des enga- 
gés dont la profession s'accompagne du nom et de la profession de leurs parents, et par l'examen des conditions de paiement du droit de contrôle.

Nous avons 63 contrats qui précisent le nom et la profession des parents. Le père est toujours un ouvrier qualifié: menuisier, tailleur d'habits, maçon, charpentier, tonnelier, etc. Il ne s'agit pas de fils de manœuvres ou de gens de service. Parmi ces 63 contrats, il y a 25 contrats de femmes. Mais ce qui nous rappelle un peu les conditions sociales de beaucoup d'engagés du XVII ${ }^{\circ}$ siècle, c'est que parmi ces 63 hommes et femmes, une vingtaine seulement ont encore leur père et mère. Une quarantaine ont perdu leur père, 25 leurs père et mère. Parmi les causes de départ il faut donc tenir le plus grand compte de l'isolement moral et matériel, de la désorientation, des difficultés de jeunes gens qui ont perdu leurs parents et de jeunes veuves.

Ajoutons la pauvreté, ou au moins la gêne d'un grand nombre. Même des gens de métier à bon salaire arrivent à bord sans le sou. Les discussions, on pourrait mieux dire les batailles, qui s'élèvent entre capitaines et engagés pour le paiement du droit de contrôle, le groupement du plus possible d'engagés sur un même acte pour avoir moins à payer, les mille autres détours pour abaisser le taux du droit, et surtout les nombreux refus de partir, le contrat signé, si l'émigrant doit payer la taxe, montrent que cette dépense de quelques sous était un gros embarras pour ces hommes qui n'avaient peut-être pas travaillé depuis quelques semaines ou qui avaient dépensé à boire leur dernier. salaire.

Pour parvenir à des comparaisons, donc à des explications, voyons si la répartition géographique des origines des engagés est la même qu'au $\mathrm{XVII}^{\mathrm{e}}$ siècle, si les agents de recrutement opèrent sur les mêmes terrains. S'il est des constances quel est leur sens ? Des cartes, des monographies sont là nécessaires, et des recherches minutieuses dans les registres paroissiaux pour serrer de près les conditions de famille et de fortune où étaient les émigrants au moment de leur départ. Ces études patientes, par paroisses ou par petites régions, comme en ont fait M. Ray- 
mond Proust à Chef-Boutonne (Deux-Sèvres) ou M. Marcel Reible pour l'Angoumois, ${ }^{42}$ sont les seules bases qui vaillent. On verra si elles révèlent des foyers très nets d'émigration.

Le premier trait remarquable est la densité des départs d'autour de La Rochelle: Aunis et Saintonge, et leur clairsemé dès qu'il s'agit de régions plus éloignées. On s'attend à ce contraste. Il est l'effet de la géographie. Une autre opposition, très apparente déjà au XVII ${ }^{e}$ siècle, est celle des origines surtout urbaines des engagés qui viennent des provinces de l'intérieur, et des origines surtout rurales des hommes qui partent des pays de l'ouest. L'arrière-pays de La Rochelle envoie surtout des campagnards. Le reste de la France surtout des ouvriers des villes. Il faut, bien entendu, tenir ici à part la ville même de La Rochelle dont les 75 engagés ne sont pas tous, tant s'en faut, même des Aunisiens.

Les engagés de Bretagne ne sont ruraux que pour la moitié, Nantes: 6 hommes; Rennes, 3; Brest: 2; Saint-Malo, Pontivy, Dinan, Vannes, Landerneau. Ni vers le Canada ni vers les Antilles les Bretons ne s'expatrient en grand nombre. Le fait est ancien.

Peu de Normands à La Rochelle: 3 hommes de Rouen ou de sa banlieue, 2 du Havre, 2 de Honfleur, 2 d'Alençon, 1 de Valognes, un de Belleville, un de Ferrière-sur-Eure, deux du diocèse de Séez, et un de Landize au diocèse de Bayeux; ce qui fait 10 engagés d'origine urbaine en face de 5 ruraux.

Même en joignant au Val-de-Loire, les plateaux du Nord et du Sud de la Loire on ne groupe que quelques noms, de moins en moins dispersés quand on se rapproche de Nantes: Orléans 1, Blois 1, Tours 7, Saumur 2, Angers 6; d'Anjou, 18 noms, de Beaupréau, Bouchoux, Bouzillée, Brain, Charny, Saint-Florentle-Vieil, Saint-Silvain, Courcelles près La Flèche, Chollet - 3 du Mans, 2 de Laval, 2 de Château-Gonthier, etc. ... 15 noms Manceaux dont 9 d'origine urbaine.

42 R. Proust, «Les engagés protestants de Chef-Boutonne ou les difficultés de l'histoire sociale », Bulletin Soc. Antiquaires Ouest, (1955) : 273287; M. Reible, «Les engagés de l'Angoumois au XVII' siècle et la question de l'émigration coloniale protestante 》 (à paraître). 
De Paris, 15 engagés, dont pour 3 seulement on nous dit la paroisse: Saint-Laurent, Saint-Sulpice, Saint-Nicolas-desChamps, Paroisses de faubourg. Presque personne de la région parisienne, même prise au sens large: Rambouillet, Montléry, La Fontaine-Ladion, du pays chartrain, Meaux, Ferrières, en «la province de Sens ».

Un Lorrain de Lunéville, un Alsacien de Landau, un Comtois de Prigué, cinq Bourguignons sont seuls à venir du Centre-Est; ce n'est pas plus que de la Savoie lointaine et étrangère.

Du Nord de Paris, en entendant par là la Picardie, le Beauvaisis, l'Artois, la Flandre, le Cambrésis et même le Hainault, viennent des gens des villes, de Beauvais, Douai, Landrecies, Saint-Omer, Cambrai, Valenciennes, Ardres, etc.

Le centre n'a jamais été très colonial: Montargis 1, Ganat 1, Moulins 2, La Charité-sur-Loire, Lyon 2, et 3 Auvergnats. Malgré les travaux importants de fortification, point de maçons marchois et seulement deux Marchois, six Limousins, dont 4 de Limoges; du Périgord, du Rouergue et du Quercy, quelques isolés, et 4 Berrichons.

Le Bordelais, la Guyenne et la Gascogne d'où partaient à la même époque tant de marchands et de jeunes gens pour les Antilles, sont aussi fort réticents et le Languedoc: Bordeaux, 5 hommes, Libourne 1, Blaye 2, Bègles, Castillon, Villeneuve d'Agenais, Valence, Duras, Salat, Mirepoix, Mont-de-Marsan, Albi.

De la région pyrénéenne, même comprise au sens le plus large, un homme ici ou là. Personne de la Bigorre. Quatre Canadiens ne trouvent pas d'autre moyen pour rentrer chez eux que de s'engager pour trois ans. Trois sont de Québec, un de Louisbourg. Peu d'étrangers : cinq Savoyards, deux marins portugais, un Irlandais seulement, un homme de Pondichéry.

On peut penser qu'une analyse plus précise de l'origine des engagés partis de l'arrière-pays - au sens large - de La Rochelle, éclairerait un peu les causes de départ. Examinons de 
plus près les lieux de départ des engagés de l'Angoumois, de la Saintonge, de l'Aunis et du Poitou.

L'Angoumois n'est point forte terre d'émigration vers le Canada, mais son émigration est en majorité urbaine et est très concentrée: 7 hommes de Cognac, 4 d'Angoulême, 5 de Barbezieux ou environs, 1 de Ruffec, 1 de Villefagnan, 1 de Marsillac, 1 de Cherves, 1 de Garat, 1 de Criteuil, 1 de Coulgens, 1 de Montagnac, 1 de Brie-sur-Matha, 1 de Saint-Barthélemy, de Vars, de Chadurie. Les quelques précisions qu'on nous donne sur les paroisses d'origine à Angoulême, qui sont paroisses de faubourg, font tourner les yeux vers une émigration de petites gens. La partie septentrionale de l'Angoumois, Ruffec et Villefagnan, qui ont fourni tant d'hommes aux îles au XVII ${ }^{\circ}$ siècle et qui en fournissent encore beaucoup au XVIII ${ }^{\mathbf{e}}$ siècle, n'a que deux isolés pour le Canada au XVIII' siècle. C'est une région à forte minorité protestante. On est porté à penser que les départs de la fin du $\mathrm{XVII}^{\mathrm{e}}$ siècle avaient pour cause les luttes et persécutions religieuses.

En tenant toujours à part La Rochelle où viennent travailler et d'où partent tant d'ouvriers de toutes les régions françaises, on n'enregistre pour l'Aunis qu'une cinquantaine de noms venant surtout des paroisses proches du port, d'Esnandes, 4 noms; de Nieul-sur-Mer, 3; de Dampierre, 5; de la Croix-Chapeau, 3; de Tangon-la-Ronde, 3. Plus loin c'est un semis diffus. Au reste, dans cette zone voisine de La Rochelle l'attraction directe du port, son mouvement d'hommes et d'affaires nous cachent les vraies causes de départ.

Les foyers les plus actifs sont dans l'île de Ré, comme au XVII ${ }^{e}$ siècle. De La Flotte 4 hommes, 3 du Bois, 3 de La Couarde, 3 de Saint-Martin, 1 de Sainte-Marie; 4 de l'île de Ré, sans autre précision, 18 au total. On avait relevé 38 noms de Rhétais pour le Canada, entre 1634 et 1714, en 80 ans. De 1714 à 1758, 18 noms en 44 ans ne sont pas une diminution sensible. De l'île d'Oléron 2 hommes. Au XVII ${ }^{e}$ 9. Elle a toujours donné moins de marins et de colons.

L'émigration saintongeaise à laquelle il faut rattacher l'île d'Oléron, part des villes et des gros bourgs: Saintes, 3 noms, 
Rochefort 2, Soubise 4, Jarnac 2, Marennes 2, Pons 1, Montendre 1, Burie 1, Brossac, Chérac, Cherbonnière, Clion, Chollet, Lonzignac, Muron. Les caractères de l'émigration saintongeaise n'ont pas changé depuis le XVII ${ }^{e}$ siècle.

La carte des lieux d'origine des engagés poitevins révèle un grand contraste entre le Poitou de l'Est ou Haut-Poitou, et les pays de Gâtine ou Poitou Central. Poitiers, écarté, qui offre neuf noms d'engagés, le Haut-Poitou a peu d'hommes. Personne de Loudun, de Châtellerault et de Niort, ce qui est assez pour nous surprendre et seulement un engagé de Vouillé, 43 un de Chauvigny, un de La Roche-Posay, un de Pouillé, ${ }^{44}$ un de Véniers près Montmorillon (?). Civray qui a toujours eu des relations suivies avec les Antilles, a trois engagés pour la Nouvelle-France. Nous connaissons la paroisse d'origine de six des neuf hommes de Poitiers: deux sont de la paroisse Sainte-Radegonde, deux de celle de Saint-Pierre, 1 de Saint-Porchaire et l'un de Saint-Didier. Il serait donc parti surtout des hommes des quartiers pauvres de la basse-ville.

C'est le Poitou Central, la Gâtine et ses abords, la région que l'on pourrait appeler le Moyen-Poitou, qui présente le semis le plus serré, semis rural essentiellement: Saint-Maixent, 4 ; Bressuire, 1; Parthenay, 1; Thouars, 3; Saint-Paul-de-Gâtine, 1; La Chapelle-Théreuil, Saint-Martin près Ré, La Mothe SaintHéraye, 3 ; Nieul-sur-l'Autige, Vouvant, Cerizay, les Herbiers, Mouchamps, Chantonnay, Coulonges-les-Royaux, le Poiré-sur-laRoche, Beaulieu-sous-la-Roche, et vers Melle: Celles, 6, SaintMartin près Chef-Boutonne, Brioux 2; l'Hermenault et SaintJulien-Lars, près Sainte-Hermine: 2 .

La Plaine, le Marais et la côte ne sont pas pays d'émigration vers le Canada, ni les Marches Communes de la Bretagne et Fontenay-le-Comte 3, Sigournay 1, Avrille 1, Montaigu, SaintGeorges de Montaigu, Olonne, etc.

Il est difficile de préciser au XVIII ${ }^{\mathrm{e}}$ siècle un parallèle entre ces lieux d'origine et les noyaux protestants que l'on pouvait

43 Encore y a-t-il deux Vouillé en Poitou, dont l'un en Bas-Poitou.

44 De même pour Pouillé. 
relever à la fin du XVII ${ }^{e}$ siècle. Les effets de la Révocation et des missions des Pères de Saint-Laurent ont supprimé ou réduit bien des centres protestants. Il n'en reste pas moins apparent que ce sont les mêmes petits pays qu'au XVII ${ }^{\mathrm{e}}$ siècle qui en gros ont des engagés. Il y a une stabilité indéniable dans les foyers de départ depuis le milieu du $\mathrm{XVII}^{\mathrm{e}}$ siècle jusqu'au milieu du $\mathrm{XVIII}^{\mathrm{e}}$ siècle, et aussi un certain rapport entre les régions d'agriculture pauvre des pays de Gâtine et de Bocage et les zones d'émigration. Mais ce rapport il ne faut pas le serrer de trop près.

Au demeurant n'oublions pas que dans la mesure, très grande croyons-nous, où cette émigration vers le Canada est au XVIII ${ }^{\mathrm{e}}$ siècle un mouvement dirigé, elle échappe à nos soucis d'analyse minutieuse. A nous aussi le jeu des offres, les belles paroles des recruteurs, mettent un écran sur les yeux.

M. Gaucher, M. Delafosse et G. Debien

Nous nous excusons auprès de nos lecteurs de ne pas leur fournir, comme d'habitude, la rubrique DOCUMENTS INÉDITS, faute d'espace, hélas. Dans notre prochaine livraison, on pourra lire, de M. Robert Le Blant, Notes sur Jean de Garibal, associé de Notre-Dame-de-Montréal vers le 24 juillet 1619 - 17 juillet 1667. 